\title{
SLE Induced Neuropsychiatric Manifestations: A unique case report
}

\section{Ryan Cordero ${ }^{1}$, Panayotis Jusakos ${ }^{2}$, Abdul Mohit ${ }^{3 *}$}

${ }^{1}$ MS4 St. George's University School of Medicine, Brooklyn, NY, USA.

${ }^{2}$ St. George's University School of Medicine Brooklyn NY, USA

${ }^{3}$ Medical Director Adult in Patient Psychiatry Department Kings County Hospital Center, New York City, USA.

*Corresponding Author: Abdul Mohit, Medical Director Adult in Patient Psychiatry Department Kings County Hospital Center, New York City, USA.

Received date: 29 July 2021; Accepted date: 09 August 2021; Published date: 13 August 2021

Citation: Cordero R, Jusakos P, Mohit A (2021) SLE Induced Neuropsychiatric Manifestations: A unique case report. J Med Case Rep Case Series 2(12): https://doi.org/10.38207/jmcrcs2021/0212110

Copyright: (C) 2021 Abdul Mohit. This is an open-access article distributed under the terms of the Creative Commons Attribution License, which permits unrestricted use, distribution, and reproduction in any medium, provided the original author and source are credited.

\begin{abstract}
This is the case of a 30-year-old African American Male, with no previous psychiatric history prior to being diagnosed with SLE in 2013, and intermittent marijuana use, presenting with an episode of psychosis. Pts psychosis has been exhibited in the form of auditory command hallucinations telling the pt to harm himself, paranoid delusions, bizarre beliefs in "spiritual things and voodoo", disorganized behavior, and anxiety onset 6 weeks prior to presentation. After being diagnosed with SLE in 2013, pt has had an extensive history of medication noncompliance, which ultimately led to SLE flares that coincided with episodes of psychosis and long-term progression of chronic anxiety issues. After medical stabilization of his SLE flares, pts psychosis, and anxiety resolve until he starts to discontinue his medications, at which point the cycle repeats itself.
\end{abstract}

\section{Introduction}

This case illustrates the association of SLE flares with episodes of psychosis in patients with SLE in the absence of being medicationinduced, or otherwise undifferentiated from functionally independent

\section{Case Presentation}

This is the case of a 30-year-old African American Male, single, living with his parents, followed by ICL and ACT team, hx of marijuana use, with no prior psychiatric history prior to be diagnosed with SLE in 2013, presenting with an episode of psychosis in the form of auditory command hallucinations telling the pt to harm himself, paranoid delusions, bizarre beliefs in "spiritual things and voodoo", disorganized behavior, paranoid and grandiose delusions, and anxiety onset 6 weeks prior to presentation.

After being diagnosed with SLE in 2013, pt has had an extensive history of medication non-compliance, which ultimately led to SLE flares that coincided with episodes of psychosis and anxiety issues. After medical stabilization of his SLE flares, pts psychosis and anxiety resolve, until he starts to discontinue his medications, at which point the cycle repeats itself. His first episode of psychosis occurred in November of 2018 leading to hospitalization for lab confirmed SLE flare with typical symptoms of joint pain, neuropathy, generalized weakness along with the associated psychosis and anxiety. Pt was stabilized, and discharged on Risperidone $2 \mathrm{mg}$ PO BID, Hydrocortisone 400mg PO BID and prednisone taper.

Pt discontinued his medications shortly after this discharge, leading to another SLE flare with associated psychosis in August 2019 due to psychiatric disorders. This case highlights the importance of recognizing organic causes of psychosis caused by medical disorders such as SLE.

bizarre behavior, paranoid delusions, and threatening his family members. Pt was again medically stabilized which additionally resolved his psychosis and anxiety and discharged with instructions to be compliant with his medications. Pts last follow up appointment for his SLE was March 2021, during which he was experiencing no rheumatologic symptoms, but labs at the time confirmed active disease with $\mathrm{C} 3$ of 78, $\mathrm{C} 4$ of 6 , and positive serology of ANA, dsDNA, SSA, SSB, Sm and RNP and negative serology for anti-centromere, Sc170, and Jo. After that appointment, pt ceased showing up to any further follow-up appointments and once again per family became medication non-compliant on all of his medications which at that time were Mycophenolate 500mg PO TID, Relafen 750mg PO BID, Hydroxychloroquine 200mg PO QD and Prednisone 20mg PO QD, Risperidone 2mg PO BID, and plan for monthly Benlysta infusions. Pt was initially evaluated in the psychiatric emergency room and eventually admitted to the adult in-patient psychiatric unit for further evaluation and plan of care management with internal medicine following along with rheumatology consultation. Labs were ordered as well as imaging studies to determine if the pt was experiencing an acute SLE flare in addition to the associated psychosis as had been seen in past admissions. Pt was initially very agitated, aggressive, 
anxious, and refusing to take all scheduled medications including medications related to his diagnosis of SLE. Pt initially exhibited paranoid and grandiose delusions, but as his time in the unit

\section{Discussion}

Systemic Lupus Erythematosus is an autoimmune chronic inflammatory condition affecting the connective tissue of multiple organ systems. Neuropsychiatric manifestations are present in around $2 / 3$ of patients with SLE [2]. These manifestations range from cognitive deficits, seizures, psychosis, peripheral neuropathy, or cerebral vascular events. Of these manifestations, psychosis only represents around $12 \%$ of the time but when it does, can be independent of typical clinical manifestations of SLE such as polyarthralgia, generalized weakness, or neuropathy [3]. It may also occur independently of another lab, neuropsychological, or imaging features typically seen during SLE flares [3]. The criteria as defined by ICD-10 for NPSLE are as follows; (ICD F06.2) [4]

1.Evidence of cerebral disease, damage, or dysfunction or of a systemic physical disease, known to be associated with one of the listed syndromes.

2.A temporal relationship (weeks or a few months) between the development of the underlying disease and the onset of the mental syndrome.

3. Recovery from the mental disorder following removal or

\section{Conclusion}

Great care must be made when evaluating patients with psychosis. Differentiating organic causes of psychosis from other etiologies of psychosis such as medication-induced, delirium, or strictly functional psychosis is of the utmost importance. Depending on the cause of psychosis, treatment plans as well as possible prophylactic measures can be adjusted accordingly to specifically target the pts condition for maximum efficacy and prevent further deterioration and delay in the appropriate treatment. In the case of NPSLE, pts is more readily prone

\section{References}

1. Nayak RB, Bhogale GS, Patil NM, Chate SS (2012) Psychosis in patients with systemic lupus erythematosus. Indian J Psychol Med. 34(1): 90-93

2. Brey RL, Holliday SL, Saklad AR, Navarrete MG, HermosilloRomo D, et al. (2002) Neuropsychiatric syndromes in lupus: prevalence using standardized definitions. Neurology. 58(8): 1214-1220.

3. Monov S, Monova D (2008) Classification criteria for neuropsychiatric systemic lupus erythematosus: do they need a discussion? Hippokratia. 12(2): 103-107.

4. World Health Organization. Geneva. Delhi: A.I.T.B.S publishers and distributors (regd.); 2002. The ICD-10 classification of
Journal of Medical Case Reports and Case Series 6 ISSN: 2692-9880 progressed, pt became more willing to take his scheduled medications regularly.

improvement of the underlying presumed cause; and

4. Absence of evidence to suggest an alternative cause of the mental syndrome (such as a strong family history or precipitating stress).

In this case, there were no apparent significant stressors apart from exacerbation of SLE due to medication non-compliance along with an absence of a family history of psychiatric illness. On the detailed assessment of the case, the patient did not exhibit any features seen with delirium such as impairment of consciousness and attention, global disturbance of cognition, and disturbance of sleep-wake cycle. Medication-induced psychosis was also ruled out because the pt was non-compliant with all of his medications during this current episode and his past NPSLE episode. Those medications such as Hydroxychloroquine and Glucocorticoids, are known to cause psychosis but are not a factor due to his medication non-compliance. Findings of NPSLE on MRI of the brain is not very reliable and usually unremarkable [5]. A frequently cited study showed evidence in SLE patients with a history of neuropsychiatric symptoms of grey matter changes which raises the possibility that grey matter alterations may be a pathological cause of psychosis in SLE patients [6].

to CNS side effects, so antipsychotics with the least potential for EPS symptoms tend to be more beneficial such as Quetiapine or Olanzapine among others. As with any pt dealing with a chronic condition, especially those that induce psychosis, medication compliance is a necessity and encouragement to adhere to schedule medications must occur to prevent any relapse of the pts more typical SLE symptoms, as well as the psychosis, observed in this case.

mental and behavioral disorders- clinical descriptions and diagnostic guidelines

5. Huizinga TW, Steens SC, van Buchem MA (2001) Imaging modalities in central nervous system systemic lupus erythematosus. Curr Opin Rheumatol.13(5): 383-388.

6. Steens SCA, Admiraal-Behloul F, Bosma Th GP, SteupBeekman GM, Olofsen H, et al. ( 2004) Selective gray matter damage in neuropsychiatric lupus. Arthritis Rheum. 50(9): 2877-2881.

7. Lieberman JA, Stroup TS, McEvoy JP, Swartz MS, Rosenheck RA, et al. ( 2005) Effectiveness of antipsychotic drugs in patients with chronic schizophrenia. N Engl J Med. 353(12): 1209-1223. 